\title{
The Paroxetine 352 Bipolar Study Revisited: Deconstruction of Corporate and Academic Misconduct
}

\author{
Jay D. Amsterdami and Leemon B. McHenryii
}

\begin{abstract}
Medical ghostwriting is the practice in which pharmaceutical companies engage an outside writer to draft a manuscript submitted for publication in the names of "honorary authors," typically academic key opinion leaders. Using newly-posted documents from paroxetine litigation, we show how the use of ghostwriters and key opinion leaders contributed to the publication of a medical journal article containing manipulated outcome data to favor the proprietary medication. The article was ghostwritten and managed by SmithKline Beecham, now GlaxoSmithKline (GSK) and Scientific Therapeutics Information, Inc. without acknowledging their contribution in the published article. The named authors with financial ties to GSK had little or no direct involvement in the paroxetine 352 bipolar trial results and most had not reviewed any of the manuscript drafts. The manuscript was originally rejected by peer review; however, its ultimate acceptance to the American Journal of Psychiatry was facilitated by the journal editor who also had financial ties to GSK. Thus, GSK was able to take an under-powered and non-informative trial with negative results and present it as a positive marketing vehicle for off-label promotion of paroxetine for bipolar depression. In addition to the commercial spin of paroxetine efficacy, important protocol-designated safety data were unreported that may have shown paroxetine to produce potentially harmful adverse events.
\end{abstract}

\section{Introduction}

Since the late 1990s, industry-sponsored ghostwriting for the medical literature has been exposed in a number of articles, plaintiffs' lawsuits and government investigations. Ghostwritten articles have been the main vehicle by which the pharmaceutical and medical device industries engage in the deliberate misrepresentation of efficacy and safety for product promotion and legal defense. ${ }^{1,2}$

In this regard, Sally K. Laden was one of the most prolific ghostwriters of the now-defunct medical communication company, Scientific Therapeutics Information (STI). A recently-posted document describes a marketing and publication plan by SmithKline Beecham, now GlaxoSmithKline (GSK) that commissioned STI to produce a manuscript for paroxetine treatment of panic disorder. In her correspondence with GSK managers, Laden wrote: "There are some data that no amount of spin will fix...." 3 On the other hand, substantial data appeared in numerous articles ghostwritten by Sally Laden such as the paroxetine 329 adolescent depression trial, the paroxetine
352 bipolar depression trial and a special issue of Psychopharmacology Bulletin "Advancing the Treatment of Mood and Anxiety Disorders: The First 10 Years' Experience with Paroxetine." ${ }^{1,4-5}$ If Laden is acknowledged at all for having produced these manuscripts, she is mentioned only in the fine print, as in the case of study 329, for "editorial assistance." 6 These and other ghostwritten publications for GSK are listed among over 100 projects that GSK commissioned from STI in a document entitled, "PaxilFunded Publications 1998 to Current," subpoenaed in 2008 in the case of Burdick vS. GSK. In addition to ghostwriting articles and letters to the editor, STI ghostwriters also prepared continuing medical education programs, conference posters, speaker training materials, and slide kits. For example, project \#1305, Nemeroff study \#352, describes the project as follows: "Write up of a clinical study. STI provided editorial assistance to primary author: writing/editing, coordinating materials needed for journal submission."7 Another item on this document is project \#1112, "Handbook: Psychopharmacology, [...] developed for clinical practitioners; overall editor C. Nemeroff," which was

\footnotetext{
i Depression Research Unit, Department of Psychiatry, University of Pennsylvania Perelman School of Medicine, Philadelphia, PA, USA (jamsterd@pennmedicine.upenn.edu)

ii Department of Philosophy, California State University, Northridge, CA, USA (leemon.mchenry@csun.edu)
}

Received: August 2019

Accepted for Publication: October 2019

Published Online: November 2019

(C) 2019 by the author(s). This article is distributed under the terms and conditions of the Creative Commons Attribution (CC BY) license. 
published as a textbook entitled Recognition and Treatment of Psychiatric Disorders: A Psychopharmacology Handbook for Primary Care under the authorship of Charles B. Nemeroff and Alan F. Schatzberg. It is hotly disputed as to whether this textbook was ghostwritten by STI. ${ }^{8}$

"Editorial assistance" was the euphemistic term STI used to describe ghostwriting. If, however, it were merely a matter of editorial assistance in the technical write up of clinical trial results, there would be no issue with what has become common practice. The problem, as we will demonstrate below, is concealing corporate input that misrepresents the data for marketing purposes.

As a result of newly-publicized documents that provide significant evidence of the misreporting of clinical trial results, this case study is a follow-up to our previous article, "The paroxetine 352 bipolar trial: A study in medical ghostwriting," published in the International Journal of Risk and Safety in Medicine..$^{5}$ In that article we critically evaluated the paroxetine 352 bipolar trial in order to demonstrate how the published report conflated primary and secondary outcome analyses, turning negative results into positive results-with conclusions that could adversely affect patient health. In the current deconstruction article, we examined forty-two publicly-available documents produced by Scientific Therapeutics Information, Inc. from paroxetine litigation. Forty of the documents were produced as part of In Re: Paxil, C.P. Ct. PA (On-Drug) and concern the production of the report of paroxetine study 352. The remaining two documents were related to the publication plans for paroxetine and another clinical trial, paroxetine study 222 for panic disorder. The documents are posted on the Drug Industry Document Archive (DIDA) web site at the University of California San Francisco.

\section{The Paroxetine 352 Study and its Findings}

Study 352, entitled "Double-Blind, Placebo-Controlled Comparison of Imipramine and Paroxetine in the Treatment of Bipolar Depression," was published in the American Journal of Psychiatry in June 2001 under the byline of Nemeroff et al. (2001). ${ }^{9}$ It was designed as an 18-site, 10week, randomized, double-blind, placebo-controlled comparison of paroxetine versus imipramine in subjects with bipolar type I major depression unresponsive to lithium carbonate at therapeutic plasma lithium levels. It had a projected study duration of 2 years. Its protocol-designated objective was to compare the efficacy and safety of paroxetine and imipramine to placebo in the treatment of bipolar depression in subjects stabilized on lithium therapy.

The primary efficacy measures were the change from baseline Hamilton Rating Scale for Depression (HRSD) total score, and the change from baseline in the Clinical Global Impression Severity (CGI/S) score for paroxetine versus placebo and for imipramine versus placebo. ${ }^{9-11}$ The comparison of primary interest was paroxetine versus placebo irrespective of baseline lithium level stratification. Protocol-stipulated secondary outcomes included the proportion of subjects with a final HRSD score $\leq 7$ or a final $\mathrm{CGI} / \mathrm{S}$ score $\leq 2$ as well as the proportion of subjects with adverse events, premature treatment discontinuation and manic or hypomanic symptoms as measured by the DSM-IIIR Mania/Hypomania Assessment and the Young Mania Rating Scale (YMRS). ${ }^{12}$ Analyses were to be performed on the entire subject population and on subjects who experienced a manic or hypomanic episode (versus those who did not). The YMRS measure was to be used to assess severity of manic and hypomanic symptoms across treatment groups, and the relationship between change in YMRS scores and HRSD scores was to be examined.

The study population consisted of outpatient subjects $\geq$ 18 years old with a history of $\geq$ one prior manic or depressive episode within the preceding 5 years. The original protocol called for a sample size of 62 subjects per treatment condition (i.e., totaling 186 subjects). However, due to poor subject enrollment, only 117 subjects were recruited into the study, resulting in a final sample size distribution of paroxetine $(n=35)$, imipramine $(n=39)$, and placebo $(n=43)$. As a result, the paroxetine 352 study was a non-informative trial with insufficient statistical power to show anything other than inconclusive results. There was no evidence of paroxetine or imipramine efficacy versus placebo in bipolar major depression, and the presentation of safety data were insufficient to draw any clinically meaningful conclusions. Despite the protocol stipulations, Nemeroff et al. failed to mention that the YMRS rating was employed as an outcome measure in the 352 study and most other manic and hypomanic safety ratings obtained with the YMRS measure were omitted from the published article. As a result, the published article inaccurately reported that there was no evidence of any paroxetine-induced manic and/or hypomanic symptoms in bipolar major depressive disorder while the suppression of safety data from the YMRS outcome measure hid the presence of possible paroxetineinduced harm.

\section{Complaint of Research Misconduct in the 352 Study}

In July 2011, a Complaint of Scientific Misconduct was filed with the Office of Research Integrity (ORI) of the Department of Health and Human Services against Dwight L. Evans, Laszlo Gyulai, Charles B. Nemeroff, Gary S. Sachs, Charles L. Bowden et al. ${ }^{13}$ As part of the formal adjudication process of the ORI misconduct case, the University of Pennsylvania organized a formal ORI Inquiry Committee comprised of three professors from the School of Medicine to investigate the allegations of misconduct. 
According to the complaint, the paroxetine 352 article was ghostwritten by employees of GSK and STI. It was published by the American Journal of Psychiatry in June 2001 under the author byline of Nemeroff et al. without acknowledging the role of GSK or the STI ghostwriters. The complaint alleged: ${ }^{14}$

(1) "that Dr. Evans and Dr. Gyulai allowed their names to be appended to a manuscript drafted by a medical communications company and, thereby, Dr. Evans and Dr. Gyulai were not legitimate authors of the manuscript" (p.3);

(2) "that the manuscript was 'ghostwritten' by STI and that the authors of the published manuscript failed to appropriately acknowledge STI's contribution" (p.3);

(3) "the preliminary drafts of the study 352 article were conceptualized and drafted by STI and not by any of the named authors" (p.5);

(4) "Dr. Evans' contribution to the preparation of the manuscript was limited to his commenting on, and approving, STI and GSK ghostwritten drafts of a manuscript on which he was designated as second author, and of which he had no direct knowledge of the accuracy of the data analyses, data interpretation (i.e., the inclusion or exclusion of particular data analyses related to safety and efficacy), or the accuracy, or the information that was written in the manuscript (by the ghostwriters)" (p.5);

(5) "Dr. Evans has been engaged in lending his name to ghostwritten articles with the same 'ghosts' (i. e., Sally Laden) at STI beginning in 1997 to at least 2003" (p.6);

(6) "[Dr. Amsterdam] was intentionally left off from the review of the data and the drafting of the manuscript because the study sponsor, GSK, and the other 'authors' knew Dr. Amsterdam's professional ethics would not allow him to lend his name to a ghostwritten work, and most importantly, his morals would not allow the alteration and manipulation of data and would not allow the other 'authors' to turn a failed study into an undisclosed promotional marketing manuscript for the sponsor" (p.7);

(7) "despite STI's significant role in the preparation and drafting of the manuscript, the final published article makes no mention of STI's role in the article and does not mention that three of its authors, Ivan P. Gergel, M.D., M.B.A., Rosemary Oakes, M.S. and Cornelius Pitts, RPh. are GSK employees." (p. 8);

(8) "Dr. Nemeroff and Dr. Evans had very little, if any, direct input into the daily conduct of the 352 study, and certainly not enough to warrant being listed as the first and second authors on a manuscript published in one of the world's leading medical journals. Rather, their positions as authors on the manuscript were solely determined by GSK for the purpose of appending the names of 'key opinion leaders' to the manuscript for marketing and commercial promotion of paroxetine" (p.10-11).

In response to these allegations of ghostwriting and plagiarism, the University of Pennsylvania and the academic authors insisted that there was no involvement with any ghostwriters and that the allegations of research misconduct were unfounded. ${ }^{14}$ The academic authors insisted that they had personally participated in the drafting of the first 352 manuscript. For example, an email press release by the University's spokesperson, Susan Phillips, indicated that an inquiry into the misconduct allegations "clearly concluded that this was not a case of ghostwriting or plagiarism." ${ }^{14}$ Likewise, in a public press statement, Dr. Evans (who was second author on the published article and Chair of the Department of Psychiatry at Penn) wrote: "After a thorough review, the inquiry concluded that each and every allegation lacked substance and credibility. ${ }^{14}$ In a similar fashion, Dr. Nemeroff (who was first author on the published article and Chair of Psychiatry at the University of Miami) reportedly told Nature Magazine that, while he was aware of STI's involvement in the preparation of the 352 manuscript, "All Sally Laden did was help collate all the different authors' comments and help with references. We wrote the paper." 14 In contrast to Nemeroff's assertion, Dr. Gary Sachs (fourth author on the manuscript and Professor of Psychiatry at Harvard) told the Boston Globe that he was "perplexed" by the allegations of ghostwriting and wrote in an email to the reporter: "When the data became available, I went to Philadelphia to help Dr. Gyulai [third author on the published article and Associate Professor of Psychiatry at Penn] draft the manuscript. We started with a blank page."14 Sachs also told Science Insider that he did not know that STI ghostwriters were involved with the manuscript preparation. Finally, Dr. Charles Bowden (fifth author on the published manuscript and Professor of Psychiatry at the University of Texas Health Science Center) stated: "I never had any sense that the manuscript was 'ghostwritten."'14

\section{Deconstruction of the Ghostwritten 352 Manuscript}

On March 13, 1997, the first draft of the 352 manuscript, prepared by Grace Johnson and Sally Laden of STI, was sent to Dr. Muriel Young, Medical Director at GSK. The cover page of the manuscript contained no author names. ${ }^{15}$ By early April 1997, GSK senior managers leading the project appeared to have made certain alterations to the content of the first manuscript draft and these revisions were now incorporated into the second manuscript draft. An email from Sally Laden to Dr. Young at GSK dated April 4, 1997, stated: ${ }^{16}$ 
We are pleased to enclosed Draft II of the manuscript "A Double-Blind, Placebo-Controlled Comparison of Imipramine and Paroxetine in the Treatment of Bipolar Depression." The manuscript has been modified based on your comments and those of Ivan Gergel, Cornelius Pitts, and Rosemary Oakes. Please note that some of Dr. Gergel's comments in the results section have been addressed in the discussion. We have also included one set of references as per Dr. Gergel's request.

Dr. Gergel has confirmed that the American Journal of Psychiatry is the target journal for publication. The manuscript will be styled according to the journal specifications for the submission draft.

We will contact the named authors (e.g., Laszlo Gyulai, Gary Sachs) once we receive your approval. At that time, Draft II of the manuscript will be sent immediately to Drs. Gyulai and Sachs for their review.

We look forward to receiving your comments on Draft II of the manuscript by April 25, 1997. We will incorporate your comments and those of the named authors and submit Draft III for your review on May 2, 1997.

This document indicates that the second manuscript draft still had no author names listed on its cover page and that the manuscript contained only GSK-directed revisions to draft \#1. The Laden email was only sent to GSK managers for their review. This document also indicates that Drs. Gyulai (of Penn) and Sachs (of Harvard) were likely designated by GSK as the original lead authors on the 352 manuscript. Thus, the first two manuscript drafts were entirely ghostwritten under the direction of GSK, without any contribution from named academic authors. Finally, the document suggests that none of the academic authors ever saw manuscript draft \#1 or \#2, until draft \#2 revisions were completed and verified by GSK.

In a subsequent May 1997 correspondence, Cornelius Pitts and Ivan Gergel of GSK provided to the STI ghostwriters their hand-written revisions to manuscript draft \#2. This revised manuscript draft indicates that the presentation of the post hoc "finding" of positive paroxetine efficacy in subjects with low baseline lithium levels was solidified into the manuscript text as if it were the primary finding of the study. In fact, this was the only "positive" statistical analysis for paroxetine in the entire study and, according to the study protocol, was unnecessary and should not have been performed. This revised draft now contained the omission of any weekly mania rating analyses or mania and hypomania symptom ratings (which were specifically stipulated in the study protocol). Furthermore, without any supporting evidence, this revised draft now indicated an absence of paroxetine-induced sexual side effects and an absence of paroxetine-induced mania. The second draft solidified the total sample size of the study at 117 subjects as if this were the protocol stipulated sample size. In this regard, all information on sample size estimates was removed from this draft, and the final power estimate provided in this draft was finessed to comport with the truncated study enrollment of 117 subjects (rather than the projected 186 subjects). Finally, GSK directed the ghostwriters to revise the discussion section of the draft to lend a favorable commercial spin to paroxetine by having them indicate that paroxetine is as efficacious as imipramine when, in fact, neither of the medications was superior to placebo in any of the outcome analyses. ${ }^{17}$ Thus, for example, while draft \#2 reads: "Paroxetine and imipramine are comparable in efficacy for the treatment of bipolar depression in patients maintained on low-lithium levels," the GSK hand-written revision reads: "Paroxetine and imipramine are comparable in efficacy for the treatment of bipolar depression showing statistically significant superiority to placebo in patients maintained on low-lithium levels." ${ }^{17}$ STI-edited iterations of these GSKdirected revisions were all eventually incorporated into the published article (p.910). ${ }^{9}$

\section{Honorary Authorship of Academic Key Opinion Leaders}

A May 19, 1997 fax from Dr. Young at GSK to Grace Johnson at STI provides a revealing picture of how the ghostwriters appended the names of the GSK-designated "authors" to the developing 352 manuscript. More than 2 months after the first draft of the manuscript was produced for GSK, Dr. Young directed the STI ghostwriter to add the following author to draft \#3: ${ }^{18}$

I would like you to add the following two names as authors on the paper:

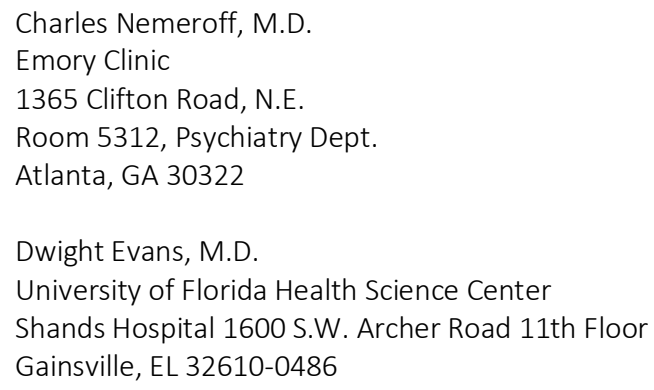

Dr. Young then goes on to direct the ghostwriter how to arrange the academic authors' names in the byline of draft \#3 and which GSK employees to add as authors. She wrote: ${ }^{18}$

The lead author should be Lazslo Gyulai, then Charles Nemeroff, Gary Sachs \& Dwight Evans, in that order. The other authors are Muriel L. Young, M.D., Ivan P. Gergel, Cornelius Pitts, \& William Bushnell. Please send a copy of the latest draft of the manuscript to Drs. Nemeroff and Evans. 
Thus, this document shows that GSK directed the ghostwriters to add Nemeroff and Evans as authors to the manuscript after two drafts had already been produced and revised by GSK. This GSK-designated order of author names would eventually change over time from draft \#3 to the final manuscript draft. For example, it is noteworthy that, at this stage in the manuscript development, there is no mention of Dr. Charles Boden as an "author" (although his name will appear on later manuscript drafts and on the published article). Moreover, with the exception of Drs. Gyulai and Sachs (who were initially designated by GSK as lead "authors" after draft \#2 was completed), the remaining academic "authors" only had their names appended to the author byline after draft \#3 was completed. To this end, on May 20, 1997, Grace Johnson of STI confirmed to Dr. Young that letters of invitation had indeed been sent to Drs. Nemeroff and Evans, inviting them to be listed as "authors" on the 352 manuscript. For example, Grace Johnson wrote: ${ }^{19}$

\section{Dear Dr. Evans:}

By way of introduction, my name is Grace Johnson, and I am an editor at Scientific Therapeutics Information, Inc (STI). STI is working with Dr. Muriel Young at SmithKline Beecham (SB) Pharmaceuticals to develop a manuscript "A Double-Blind, Placebo-Controlled Comparison of Imipramine and Paroxetine in the Treatment of Bipolar Depression."

We are pleased to invite you to participate as an author on this article. Enclosed please find Draft II of the manuscript for your review. You may mark your comments directly on the manuscript. Please return your comments on Draft II of the manuscript by June 12, 1997. We will incorporate your comments and those of the other reviewers and submit Draft III for your review. Following the approval of Draft III, the manuscript will be styled for submission to the American Journal of Psychiatry.

Please do not hesitate to contact us with any questions or comments during your review. We look forward to working with you on this project and to submitting the manuscript for publication.

Thus, despite their later, public assertions that the 352 article was not ghostwritten, it is clear to us that Dr. Evans and Dr. Nemeroff were definitely aware of the existence of STI's involvement in the production of the manuscript. It is also clear to us that their names were being appended as "honorary" or "guest" authors on the third draft of the developing manuscript to which they had made no author contributions.

A subsequent, internal June 6, 1997 STI memorandum from Grace Johnson to her STI associates summarized the current status of the 352 manuscript project: ${ }^{20}$

$D[\mathrm{raft}] 2$ was sent to SB and Drs. Gyulai and Sachs on April 4, 1997. SB added to more authors (Dr. Nemeroff and Evans) on May 20, 1997 [sic]. To date, we have received comments from Dr, Young, Neil Pitts, and William Bushnell. Dr. Gyulai requested SB to complete additional statistical analysis on the data. So, D[raft] 2 of the manuscript is currently on hold until the analysis is complete. The proposal states four drafts. However, with so many reviewers and additional statistical analysis on $\mathrm{D}$ [raft] 2, another draft might be needed. (This may depend on if the statistical analysis changes the results section of the study.)

At this point, there were a total of 4 GSK-designated academic authors, i.e., Drs. Gyulai, Sachs, Nemeroff, and Evans, and 4 GSK employees. Dr. Bowden's name had not yet been added to the author byline. It was also noteworthy that Dr. Gyulai's request for additional post hoc statistical analyses by GSK would result in greater production costs to the sponsor (see below). Finally, this internal memorandum noted that GSK had authorized STI to develop 25 PowerPoint slides describing the results of the 352 study (presumably for marketing and educational purposes). This project was to be completed in May 1997, ahead of the publication of the 352 article. By December of 1997, it appeared that the lead author, Dr. Gyulai, had fallen into disfavor with GSK for his requisition of additional statistical analyses, which led to a delay in the completion of the 352 project.

In a December 15, 1997 letter from Sally Laden to Dr. Nemeroff, Laden indicated that Charles Bowden had now been designated by GSK as a $5^{\text {th }}$ academic author and that Dr. Gergel of GSK had advised STI that he, Dr. Nemeroff, would heretofore be designated by GSK as lead author on the 352 project. Laden wrote: ${ }^{21}$

Ivan Gergel asked me to send you the enclosed author comments on the Paxil bipolar disorder study report. STI progressed to the Draft II stage at which point Dr Gyulai has held onto the paper without response to us.

Enclosed are comments from yourself, Dwight Evans, and Charles Bowden.

Ivan mentioned that you will be leading development of this manuscript in the future, but that arrangements with $\mathrm{Dr}$ Gyulai have not yet been finalized. Once I learn more from Ivan, I will be in touch with you to discuss our next steps.

Thanks for understanding this process. Please don't hesitate to call me if you have questions or require additional information.

Four months after Laden informed Nemeroff that he would assume the lead on the 352 project, Laden sent an April 3, 1998 proposal to Dr. Gergel at GSK to formalize the designation of Nemeroff to restart the stalled 352 project. The proposal contained a detailed explanation for an additional $\$ 10,000$ cost of completing the 352 article. It further detailed the cost for every step of the ghostwriting, 
review and submission of the manuscript. The proposal stated:22

STI will develop up to four drafts: Draft I is the initial draft that we will receive from Dr Nemeroff and which we will edit and follow his direction. This will be reviewed by the sponsor and Dr Nemeroff, Comments on Draft I will be incorporated into Draft II, which will be sent to the same reviewers for review and critique. Draft III is the prejournal submission draft and will be sent to the author and sponsor for final review and approval prior to developing the journal submission package. Draft IV is the journal submission draft prepared for the journal, for which art work will be professionally drawn and the manuscript styled according to the selected journal.

It should be noted also that page 7 of this April $3^{\text {rd }}$ proposal indicates in the "Time and Events" section that SB, i.e., GSK, will receive draft 1 from Nemeroff one month after STI receives the manuscript. This statement, however, contradicts earlier STI documents (cited above). In this regard, draft \#1 had already been produced by STI on March 13, 1997. ${ }^{16,17}$ Thus, irrespective of whether Laden's April 3, 1998 designation of "draft \#1" is the same as the original draft \#3, a comparison of the two STI manuscripts makes it immediately apparent that both drafts contain the same information that is present in the original STI draft \#1 from March 13, 1997. From our reading of the documents, the most plausible interpretation is that the April 3, 1998 STI contract from Laden to Gergel did not begin afresh with a new 352 draft \#1.

\section{Role of the Journal and the Peer-Review Process}

On February 24, 1999, Sally Laden (under Dr. Nemeroff's name) began the submission process of the 352 manuscript to the American Journal of Psychiatry. Laden wrote to each GSK-designated author to obtain their signed copyright release to the journal. The "Manuscript Submission Approval and Copyright Transfer" reads: ${ }^{23}$

I have been sufficiently involved in this work to take public responsibility for its validity and final presentation as an original publication. I can and will provide documentation of my work upon reasonable request and I have fulfilled the obligations for full disclosure and authorship as described by the American Journal of Psychiatry.

In a subsequent communication from Laden to Nemeroff dated March 5, 1999, Laden informed Nemeroff that: ${ }^{24}$

After I sent what I thought was the final version (SB had given it their blessing) to all authors for their sign-off, $\mathrm{Dr}$ Gyulai pointed out that the ad-hoc analysis of high vs low HAMD patients excluded those patients with thyroid changes. SB quickly re-ran the data and found that the significant differences between imipramine and placebo and the trends for paroxetine were lost. Thus, we deleted
Figure 2 and deleted one sentence from the text. Because this wasn't the main point of the paper, I'm hoping that this change is OK. I'm assuming that it is and am sending the submission package to you today.

Thus, it appears that Laden unilaterally revised the manuscript (i.e., without any consultation from the academic or GSK authors) to avoid reporting the newlyanalyzed, post hoc "negative" finding. Thus, if Laden had not made this unilateral editorial decision, there may have been no "positive" analyses of any sort to report on paroxetine.

An email thread between Nemeroff and Laden dated July 13, 1999 that was forwarded to Cornelius Pitts at GSK provided information on the peer-review status of the 352 manuscript by the American Journal of Psychiatry. Laden wrote: ${ }^{25}$

I'm reviewing my list of projects with SB and have a few questions. 1. Have you heard from Am J Psychiatry about the bipolar paper?...

Nemeroff responds: ${ }^{25}$

I am in Zurich as I respond at midnight visiting with Roche. The answers are: 1. Jack Gorman [Deputy Editor of the American Journal of Psychiatry] told me that the Bipolar paper has come back from review and will be accepted after revisions. I haven't received the reviews yet but you will be the first to know, I promise....We love working with you.

A subsequent September 13, 1999 email from Laden to Nemeroff (copied to Pitts at GSK) provided additional information on the status of the peer-review process. Apparently, the peer-reviewed manuscript was returned from the American Journal of Psychiatry and was back on the desk of Sally Laden at STI. Laden informed Nemeroff that extensive revisions were requested by the reviewers and that many of the comments involved statistical issues that she could not address, as she (i.e., Laden) revised the peerreviewed manuscript. As a result, Laden sent the partlyrevised manuscript to Pitts at GSK to address the requested statistical revisions with the GSK statistician. Laden wrote to Nemeroff: 26

Last week, I finished with my first cut at the Am J Psych reviewers' extensive comments to this paper. Many of their comments were statistical in nature, which only the SB statistician can answer. Neil Pitts has it now and I'll stay in touch with him about the progress of their review.

A subsequent email exchange, between Laden and Pitts, dated March 15, 2000 provides more insight into the STI/GSK revisions that were made to the 352 manuscript. The revisions suggested by Pitts to Laden were memorialized in an email "note-to-file" made by Sally Laden to herself: ${ }^{27}$

Note to self:

make these changes to the bipolar manuscript 
1. emphasize that only 5 of 8 patients were in the efficacy population and this didn't come through in the manuscript revision.

2. tone it down and the efficacy of those 5 patients who had efficacy change scores was similar to the overall study.

In a subsequent April 5, 2000 email, Laden provides Nemeroff with a further update on the revisions of the 352 manuscript: ${ }^{28}$

The bipolar manuscript has been revised [by GSK] to include the statistical reanalysis, and Rocco Zanninelli and Neil Pitts have signed off on it. It is being fedexed to you tomorrow and you will receive it on Wednesday. If you approve the manuscript, I will prepare a brief response to the journal so that you can resubmit it.... Again, upon your approval to proceed, I'll have a submission package prepared for you to submit the manuscripts to the journal.

This email from Laden to Nemeroff, together with the September $13^{\text {th }}$ email exchange above, indicate to us that Nemeroff and the other academic authors had little or no scientific input into the original or revised 352 data analyses and that virtually all of the revisions were made by Sally Laden and GSK employees i.e., Drs. Zaninelli, Pitts, Oakes, and Gergel. Sally Laden, and not Nemeroff, wrote the revision cover letter to the editor of the American Journal of Psychiatry) (i.e., Jack Gorman). In a somewhat curious addendum to this email exchange, Nemeroff wrote to Laden: ${ }^{28}$

Ivan Gergel, MD who was with SKB and was a coauthor [on manuscript draft \#3] must be reinserted as a coauthor. I will not compromise on this as he was involved in the early drafts and this is simply an ethical issue. Dwight and other authors agree about this. We must take the high road here.

In an email dated May 11, 2000, Laden wrote to Dr. Evans and the other academic authors indicating that she needed their approval of the revised 352 manuscript that was prepared for Nemeroff. Laden advised the authors of the revision process: ${ }^{29}$

Significant re-analysis has been accomplished and the revisions have been made and approved by Dr Nemeroff, Rocco Zaninelli, MD (of SB), and Neil Pitts, RPh (of SB). Dr Nemeroff will be submitting the revised manuscript to the journal early next week. A copy of the revised manuscript (note that new references are not yet renumbered) and Dr Nemeroff s point-by-point rebuttal to the journal are attached in WORD files below.

Evans responded to Laden: ${ }^{29}$

Dear Sally,

Masterful! No additional changes recommended.

Dwight
An email dated June 12, 2000, indicated that further difficulties had arisen in publishing the 352 manuscript. It appears that the American Journal of Psychiatry had sent the revised manuscript to an additional, independent statistical reviewer who rejected the revised manuscript and instead recommended additional analyses, revisions and the elimination of all commercial bias. Although the official letter from Gorman to Nemeroff, printed on American Journal of Psychiatry letterhead, was not sent to Nemeroff until June 22, 2000, concerns about the manuscript were already being expressed by the STI ghostwriter and GSK. In this regard, Laden wrote to Nemeroff on June $12^{\text {th. }}{ }^{30}$

Thanks for copying me on the email from Jack [Gorman] about Am J Psychiatry's review. I've passed this along to SB and await their reaction. What are your feelings about this?

Nemeroff responded to Laden: ${ }^{30}$

Jack is clearly willing to fight for the paper. The statistician's point is well taken, namely the secondary hypothesis testing. I think we should see if the SB statisticians are able to respond adequately to the criticisms and moreover if we can tone down the perceived commercial bias so Jack can pull it over the line. If not, we can publish it relatively quickly in Depression and Anxiety. I can use the AJP reviews from the first review.

Laden replied to Nemeroff that she will pass the information about the need for additional revisions and the assistance from Jack Gorman to the GSK managers. Gorman had significant financial ties to GSK as a recognized key opinion leader, member of GSK's psychiatry advisory board, speakers' bureau and frequent spokesperson for paroxetine (Paxil) on television programs and advertisements. ${ }^{31}$

Despite Nemeroff's reassurance to Laden, it appears that GSK was, nonetheless, increasingly frustrated with the additional time, cost, and uncertainty of getting the 352 manuscript published in the American Journal of Psychiatry. To wit, an internal email among STI employees, dated June 13, 2000, revealed GSK's dissatisfaction with STI's ghost management of the 352 project. Marion Philips and Sally Laden describe to STI President (John Romankiewicz) the extent of GSK's dissatisfaction: ${ }^{32}$

Marion - this project (the bipolar paroxetine manuscript) has come into trouble with the client [i.e., GSK]. It has been a protracted process - we are currently at Draft $\mathrm{XI}$, if my calculations are correct. They are disgusted with the author (Charlie Nemeroff) and with the fact that they have to pay an agency [i.e., STI] extra to get it done. Remember, this is the client (Rocco Zaninelli [Medical Director at GSK] who refuses to work with any outside medical writers, including STI. A brief history is this:

1. Submitted to Am J Psychiatry, 
2. Journal sent back significant revisions, but no promise of acceptance

3. Many drafts later, the revised version (draft X or XI) was resubmitted

4. Yesterday, journal responded that they might consider it, but significant revision was still needed.

5. Charlie Nemeroff feels that with more revision we might get it in the Am-J Psychiatry, but that we could probably get it published in his journal (Depression \& Anxiety or the British J Psychiatry)

I advised client of this immediately. They responded by saying that they don't care where it is published, they just don't want to be charged extra. They want to turn this [i.e., the production of the 352 manuscript] over to Charlie so that he can finish it up.

Meanwhile, behind the scenes, a private email from Gorman to Nemeroff, dated June 12, 2000, detailed how Gorman could facilitate the revision and publication of the rejected 352 manuscript. Gorman indicates that he was not quick enough in forestalling the statistical reviewer from rejecting the 352 manuscript; but would nonetheless get it published, with GSK's direct assistance. Gorman wrote: ${ }^{33}$

I got the bipolar depression paper back and wanted to give you a heads up. The revision went automatically to the statistical reviewer before coming to me, which is the usual custom on resubmitted manuscripts. The statistical reviewer adamantly recommends rejection. I read it again carefully and I still think the paper is important as one of the only if not the only one of its kind. However, journals are now under fierce scrutiny about drug company sponsored studies, thanks in large part to Marcia Angel's recent stuff in the NEJM.

The paper still has a very biased tinge to it. Look, the fact is that the study was beautifully designed and the results are important. But in the high lithium level group the two active drugs did no better than placebo and it is a legitimate conclusion that raising the lithium level alone may be sufficient to treat bipolar depression. I think you would want to make that point much more strongly.

Second, using the main analyses, the ITT for all groups, there is no statistically significant drug versus placebo effect. The journal will never allow 'numerical superiority' as a standard. So the second conclusion is that for the study as a whole, drugs didn't do better than placebo.

In a secondary analysis you can show that if you don't want to raise lithium level, there is a completer finding that imipramine and paroxetine are better than placebo, but it must be labelled as a very post hoc observation.

Finally, we all know that paroxetine is better tolerated than imipramine but I don't see it in this data set. None of the comparisons for adverse side effects or drop outs is statistically significant, [and] the claim that paroxetine is better on that basis than imipramine is not warranted.
You'll get all this back in the form of an invitation to revise again. Even if I bend, no way NCA [Nancy C. Andreason, Chief Editor] would allow it in its present state so it is moot for me to go ahead. I think you might want to go over it again with the SKB people and let them know that the study just doesn't support much in the way of a claim that paroxetine worked particularly well here compared to other interventions.

If I've missed something, of course let me know. I want to be helpful and this was a lot of work and a great study.

In follow up to this June $12^{\text {th, }}$ back channel email from Gorman to Nemeroff, Laden sent a fax correspondence on July 5, 2000 to Drs. Raj Kumar and Rocco Zaninelli of GSK to reassure them that the 352 manuscript would most probably be accepted for publication by the journal editor, if a few minor cosmetic revisions were made to the manuscript. However, Laden also recognized that GSK may not want to expend additional time and money on publishing the 352 manuscript in the American Journal of Psychiatry; in which case it will definitely be accepted in Nemeroff's Depression \& Anxiety journal. ${ }^{34}$

It appears that Nemeroff was probably successful in gaining Dr. Kumar's approval to have GSK continue working with STI and Gorman. As a result, Dr. Kumar agreed to allow STI to move forward with another revision. This resulted in a July 26, 2000 letter in which Laden writes to Kumar: ${ }^{35}$

At the request of Charlie Nemeroff, I am enclosing the most recent correspondence from Am J Psychiatry related to this manuscript. Charlie tells me that you are willing to continue revising this manuscript and submit it a third time to the journal. According to Charlie, this will be accomplished as follows:

1. Rosemary Oakes will address the statistical reviewer's comments and make changes to the manuscript.

2. When done, she will send these changes to STI to be incorporated into the manuscript.

3. STI will send the revised manuscript to Charlie, who will revamp it and remove the 'marketing' tone to which the journal objected.

4. Charlie will either submit the manuscript directly or return it to STI to be restyled for resubmission to the journal.

Enclosed please find a copy of the manuscript plus the journal's reviews and correspondence.

I trust that this is an accurate summary of the agreement made to finish this project. If not, kindly contact me directly.

A subsequent October 31, 2000 email from Laden to Nemeroff details the revisions that were made by Laden and GSK to the 352 manuscript. Laden indicates that the revised 
manuscript fulfills all of Gorman's June 22, 2000 officiallyrecommended revisions. Laden wrote to Nemeroff: ${ }^{36}$

The changes consist of the addition of more statistical detail in Table 2 and greatly toned down [the commercial] text in the Abstract, Results, and Discussion about the findings.

Laden notes that: ${ }^{36}$

... this version is sufficiently noncommercial and fulfills the directives outlined by the journal.

Finally, Laden indicates to Nemeroff that if he approves the Gorman and GSK-recommended revisions, she will prepare a submission packet for Nemeroff containing: ${ }^{36}$

a detailed response to the reviewers, 4 copies of the manuscript and cover letter, a new glossy for the figure, and all files on a disk.

On November 11, 2000, Laden ghostwrote a cover letter (in Nemeroff's name) to Jack Gorman at the American Journal of Psychiatry, detailing the second revision to the 352 manuscript. ${ }^{37}$ This letter was then placed on Emory letterhead for submission to the journal via Nemeroff's office staff. The cover letter states that the report of the 352 trial: ${ }^{37}$

represents the largest placebo-controlled trial of bipolar depression ever conducted [sic], and contains important and novel information to the field.

It is noteworthy, however, that there is no mention of the study being prematurely terminated for insufficient subject enrollment or that it had insufficient power to test any of the primary or secondary study aims. It also states that the study outcomes are unique, when, in fact, the GSK authors, journal reviewers and journal editor noted that the study was a negative trial. ${ }^{24,33}$

While the peer-review and revision process of the 352 manuscript finally resulted in its acceptance for publication by the American Journal of Psychiatry on January 6, 2001, we would note that this process (such as it was) failed to adhere to the International Committee of Medical Journal Editors (ICMJE) policy regarding authorship, according to which both honorary authorship and ghost authorship are considered forms of misconduct. The American Journal of Psychiatry policy in place at the time of the submission of the Nemeroff et al. manuscript stated: ${ }^{38}$

All persons designated as authors should qualify for authorship. Each author should have participated sufficiently in the work to take public responsibility for the content.

Authorship credit should be based on substantial contributions to: 1) conception and design or analysis and interpretation of data, and 2) drafting the article or revising it critically for important intellectual content, and on 3 ) final approval of the version to be published.
Conditions 1, 2, and 3 must all be met. Participation solely in the acquisition of funding or the collection of data does not justify authorship. General supervision of the research group is also not sufficient. Any part of an article critical to its main conclusions must be the responsibility of at least one author

Only those with key responsibility for the material in the article should be listed as authors; others contributing to the work should be recognized in an Acknowledgement. Editors will require authors to justify the assignment of authorship.

On September 22, 2016, the current authors wrote to the American Journal of Psychiatry Chief editor, Robert Freedman, to request retraction of the Nemeroff et al. article on the basis of the violation of journal policy and the misrepresentation of study 352 results. Dr. Freedman did not respond, and thus the scientific record remains uncorrected. (The letter from the current authors is published as a supplemental file to this article).

\section{Discussion}

The paroxetine 352 study was a non-informative trial with insufficient statistical power to show anything but inconclusive results. There was no evidence of any paroxetine (or imipramine) efficacy relative to placebo in bipolar major depression and the suppression of safety data hid the presence of paroxetine-induced harm.

The misrepresentation of the 352 study as a published report was facilitated by STI ghostwriters working in conjunction with GSK employees and the editor of the American Journal of Psychiatry. Given the documentary evidence presented above, and despite their uniform denials to the Penn ORI Inquiry Committee, it is difficult to see how the academic authors could not have been aware that a medical writing firm was involved in the manuscript production. Moreover, most of the academic authors apparently misled the American Journal of Psychiatry by appending their signature to the "Manuscript Submission Approval and Copyright Transfer" that claimed they were sufficiently involved in the work to take public responsibility for its validity and final presentation. These academic "authors" failed to adhere to the journal's authorship criteria and they violated the policy by failing to disclose the role of STI and GSK in the development of multiple manuscript drafts.

The only mention in the published Nemeroff et al. article of drug company support is the rather misleading statement, "Supported by NIMH grant $\mathrm{MH}-51761$ and a grant from GlaxoSmith-Kline," suggesting that the primary financial support for the 352 study was provided by a NIMH supported grant with secondary support from GSK. ${ }^{9}$ This, of course, was not the case. 
From our deconstruction of the documentary evidence, it appears that none of the academic authors had access to, or reviewed, the actual patient-level data or the repeated GSK-produced data analyses and it is certainly false that they had complete freedom to direct its analysis and its reporting without influence from the sponsor. We would also note that the ethical protestation by Nemeroff, Evans and the other academic authors regarding the guest authorship of Ivan Gergel [after his departure from GSK to Forest Laboratories, Inc.] is ironic given the fact that none of the academic "authors" had any involvement in the 352 study and that they were all designated by GSK for inclusion in the author byline. It is also obvious to us that the journal editor, Jack Gorman, took unethical and unprofessional editorial license with the journal peer-review process in order to facilitate publication of the 352 manuscript. Gorman also failed to have the 352 authors justify the assignment of authorship. In our view, the authorship policy of the American Journal of Psychiatry is largely window dressing common for journals of the sort that publish industry-sponsored trials.

The ORI Inquiry Committee of the University of Pennsylvania ignored the allegations of research misconduct in the performance of the 352 study and, instead, focused on the ghostwriting aspect of the published article. In this regard, Penn failed to examine the available evidence in the case and ignored the most egregious evidence of misconduct, i.e., the newly-posted STI documents. This omission allowed the University to avoid public embarrassment and to clear their professors of any wrongdoing. Thus, instead of punishing their professors for academic misconduct, the University whitewashed the presence of any misconduct within its psychiatry faculty. Penn's conclusion of no wrongdoing by its professors provoked such a public outcry of indignation that the issue of ghostwriting at the University of Pennsylvania reached the desk of the President of the United States. ${ }^{39}$

\section{Conclusion}

Because ghostwriting is designed to evade detection and is only revealed as a result of litigation or government inquiries, it is therefore imperative to document the cases in which ghostwriting has facilitated misrepresentation of clinical trial results. The integrity of science depends on the trust placed in individual clinicians and researchers and in the peer-review system which is the foundation of a reliable body of knowledge. When academic researchers allow their names to appear on ghostwritten articles, they betray this basic ethical responsibility and are guilty of academic dishonesty. Medical journal editors are entrusted with significant power as gatekeepers of the scientific record. They also bear the responsibility to ensure that the journals are not publishing manuscripts guilty of fraud, fabrication and plagiarism. Ghostwriting is a serious problem because it is a dishonest attribution of the origin of the manuscript, it disguises marketing and public relations objectives of forprofit companies as science, conceals conflicts of interest of named "authors" on manuscripts, misrepresents the results of scientific testing, and, most importantly, has contributed to fatal consequences in cases in which the safety of drugs are misreported.

\section{Limitations}

The authors warrant that findings have been reported fairly and non-selectively. It is, however, always possible that there are gaps in the record due to inadequate response to discovery in litigation. Since the complete deconstruction of each STI document could not be exhaustively undertaken in the course of this review, we recommend that readers examine each STI document that is posted on the Drug Industry Document Archive (DIDA) web-site at the University of California San Francisco (see https://www.industrydocuments.ucsf.edu/drug/collections (paxil-litigation-documents/).

\section{Acknowledgments and Disclosure:}

This research was not supported by any federal, corporate, or private funding agency or grant. The authors thank Ronald Goldman, Esq. and Michael Baum, Esq., for legal review of the manuscript, the Drug Industry Document Archives (DIDA) for posting the paroxetine 352 study documents on their website, two anonymous reviewers from the journal and Tess Bird for helpful suggestions to the final draft. The views expressed herein are those of the authors alone and not those of any other person, firm or entity. Dr. Amsterdam received legal support from Baum, Hedlund, Aristei \& Goldman of Los Angeles, California for his Complaint of Scientific Misconduct against Dwight L. Evans, Laszlo Gyulai, Charles Nemeroff, Gary S. Sachs and Charles L. Bowden provided to the United States Department of Health and Human Services Office of Research Integrity in July 2011. Dr. McHenry has been a research consultant for Baum, Hedlund, Aristei \& Goldman since 2003.

\section{References:}

1. McHenry L. Of sophists and spin-doctors: Industrysponsored ghostwriting and the crisis of academic medicine. Mens Sana Monographs.2010;8: 129-145

2. Sismondo S. Ghost management: How much of the medical literature is shaped behind the scenes by the pharmaceutical industry? PLoS Medicine. 2007;4(9): e286.

3. STI. Email from Sally Laden to Daniel Burnham. 2000 Dec 14. Available from:

https://www.industrydocuments.ucsf.edu/drug/docs/\#id=sn $\underline{\mathrm{x} \mid 0228}$ 
4. McHenry L, Jureidini J. Industry-sponsored ghostwriting in clinical trial reporting: A case study. Accountability in Research. 2008;15: 152-167.

5. Amsterdam JD, McHenry L. The paroxetine 352 bipolar trial: A study in medical ghostwriting. International Journal of Risk and Safety in Medicine. 2012;24(4): 221-231.

6. Keller MB, Ryan ND, Strober M, Klein RG, Kutcher SP, Birmaher B, Hagino OR, Koplewicz H, Carlson GA, Clarke GN, Emslie GJ, Feinberg D, Geller G, Kusumakar V, Papatheodorou G, Sack WH, Sweeney M, Wagner KD, Weller EB, Winters NC, Oakes R, McCafferty JP. Efficacy of Paroxetine in the treatment of adolescent major depression: a Randomized, controlled trial. Journal of the American Academy of Child and Adolescent Psychiatry. 2001;40:762772.

7. STI. Paxil-funded publications 1998 to current. 2005. Available from:

https://www.industrydocuments.ucsf.edu/drug/docs/\#id=zn $\underline{x \mid 0228}$

8. Wilson D. Drug maker wrote book under 2 doctors' names, documents say. The New York Times. 2010 Nov 30: B3

9. Nemeroff CB, Evans DL, Gyulai L, Sachs GS, Bowden CL, Gergel IP, Oakes R, Pitts CD. Double-blind, placebocontrolled comparison of imipramine and paroxetine in the treatment of bipolar depression. American Journal of Psychiatry. 2001;158(6): 906-912.

10. Williams, JBW. A structured interview guide for the Hamilton Depression Rating Scale. Arch Gen Psychiat. 1988;45: 742747.

11. Guy W. ECDEU assessment manual for psychopharmacology. US Department of Health, Education, and Welfare, Public Health Service, Alcohol, Drug Abuse, and Mental Health Administration, National Institute of Mental Health, Psychopharmacology Research Branch, Division of Extramural Research Programs. 1976.

12. Young RC, Biggs JT, Ziegler VE, Meyer DA. A rating scale for mania: reliability, validity and sensitivity. Brit J Psychiat. 1978;133: 429-435.

13. Esfandiari B. Complaint of Scientific Misconduct, Office of Research Integrity of the Department of Health and Human Services. 2011. Available from:

http://psychrights.org/Research/Digest/Science4Sale/11070 8EthicsComplaintAgainstEvansGyualaiNemeroffSachsBowde $\underline{\text { netal.pdf }}$

14. Esfandiari B. Complaint of Scientific Misconduct, Office of Research Integrity of the Department of Health and Human Services. 2012. Available from: http://blogs.nature.com/news/files/2012/06/JayAmsterdam-today-filed-a-24-page-complaint-with-theOffice-of-Research-Integrity-at-the-US-National-Institutesof-Health.pdf
15. STI. STI008109, Draft I. 1997 Mar 13. Available from: https://www.industrydocuments.ucsf.edu/drug/docs/\#id=fr $\underline{\text { hk0228 }}$

16. STI. STI008106, Letter from Grace E. Johnson to Muriel L. Young. 1997 Apr 4. Available from: https://www.industrydocuments.ucsf.edu/drug/docs/\#id=fr hk0228

17. STI. STI008050, Facsimile from Neil Pitts to Grace Johnson. 1997 May 7. Available from: https://www.industrydocuments.ucsf.edu/drug/docs/\#id=gr $\underline{\text { hk0228 }}$

18. STI. STI008093, Facsimile from Muriel L. Young to Grace Johnson. 1997, May 19. Available from: https://www.industrydocuments.ucsf.edu/drug/docs/\#id=xr $\underline{\text { hk0228 }}$

19. STI. STI008090, Facsimile from Grace Johnson to Muriel Young. 1997, May, 20. Available from: https://www.industrydocuments.ucsf.edu/drug/docs/\#id=irh $\underline{\mathrm{k} 0228}$

20. STI. STI007107, Memorandum from Grace Johnson to Marion Philips. 1997, Jun 6. Available from: https://www.industrydocuments.ucsf.edu/drug/docs/\#id=kr $\underline{\mathrm{hk0228}}$

21. STI. STI007238, Letter from Sally K. Laden to Charles B. Nemeroff. 1997, Dec 15. Available from: https://www.industrydocuments.ucsf.edu/drug/docs/\#id=mr hk0228

22. STI. STI008363, Proposal for a Journal Article by Sally K. Laden and John A Romankiewicz. 1998 Apr 3. Available from: https://www.industrydocuments.ucsf.edu/drug/docs/\#id=nr $\underline{\mathrm{hk0228}}$

23. STI. STI008194, Letter from Sally K. Laden to Cornelius D. Pitts. 1999, Feb 24. Available from:

https://www.industrydocuments.ucsf.edu/drug/docs/\#id=yr hk0228

24. STI. Email from Sally Laden to Charlie Nemeroff. 1999, Mar 5. Available from: https://www.industrydocuments.ucsf.edu/drug/docs/\#id=rr $\underline{\mathrm{hk0228}}$

25. STI. STI008029, Email from Sally Laden to Neil Pitts. 1999, Jul 14. Available from:

https://www.industrydocuments.ucsf.edu/drug/docs/\#id=sr hk0228

26. STI. STI007834, Email from Sally Laden to Charlie Nemeroff. 1999, Sep 13. Available from:

https://www.industrydocuments.ucsf.edu/drug/docs/\#id=zr $\underline{\mathrm{hk0228}}$

27. STI. STI008748, Email from Sally Laden to Cornelius D. Pitts. 2000, Mar 15. Available from: https://www.industrydocuments.ucsf.edu/drug/docs/\#id=fs $\underline{\mathrm{hk0228}}$ 
28. STI. STI008839, Email from Sally Laden to Charles Nemeroff. 2000, Mar 27. Available from:

https://www.industrydocuments.ucsf.edu/drug/docs/\#id=gs $\underline{\mathrm{hk0228}}$

29. STI. STI008941, Email from Dwight L. Evans to Sally Laden. 2000, May 11. Available from:

https://www.industrydocuments.ucsf.edu/drug/docs/\#id=hs hk0228

30. STI. STI007733, Email Sally Laden to Charles Nemeroff. 2000, Jun 12. Available from: https://www.industrydocuments.ucsf.edu/drug/docs/\#id=jsh $\underline{\mathrm{k} 0228}$

31. Koerner B. Disorders made to order. Mother Jones. 2002 Jul/Aug. Available from: http://motherjones.com/politics/2002/07/disorders-madeorder

32. STI. STI007102, Email Sally Laden to Marion Philip. 2000, Jun 13. Available from: https://www.industrydocuments.ucsf.edu/drug/docs/\#id=gt $\underline{\mathrm{hk0228}}$

33. STI. STI008644, Email Jack Matthew Gorman to Charles Nemeroff. 2000, Jun 12. Available from:

https://www.industrydocuments.ucsf.edu/drug/docs/\#id=ft $\underline{\mathrm{hkO} 228}$

34. STI. STI008633, Facsimile from Sally K. Laden to Rai Kumar. 2000, Jul 5. Available from:

https://www.industrydocuments.ucsf.edu/drug/docs/\#id=ht $\underline{\text { hk0228 }}$

35. STI. STI008623, Facsimile from Sally K. Laden to Rai Kumar. 2000, Jul 26. Available from:

https://www.industrydocuments.ucsf.edu/drug/docs/\#id=jth $\underline{\mathrm{k} 0228}$

36. STI. STI007369, Email from Sally Laden to Charlie Nemeroff. 2000, Oct 31. Available from:

https://www.industrydocuments.ucsf.edu/drug/docs/\#id=ns $\underline{\mathrm{hk0228}}$

37. STI. STI008496, Letter from Charles B. Nemeroff to Jack M. Gorman. 2000, Nov 30. Available from: https://www.industrydocuments.ucsf.edu/drug/docs/\#id=ps $\underline{\mathrm{hkO} 228}$

38. American Journal of Psychiatry. Information for Authors. American Journal of Psychiatry. 2000;157(5): A75-78.

39. Brian D. Project on Government Oversight, Letter to President Obama. 2000. Available from: https://www.pogo.org/letter/2011/07/pogos-letter-topresident-obama-on-bioethics-commission-chairsghostwriting-practices/ 